\title{
Mass Spectrometry and Analytical Chemistry
}

\author{
Fred W. McLafferty \\ Baker Chemistry Laboratory, Cornell University, Ithaca, New York, USA
}

A nalysis was once the major discipline of chemistry, and most research sought better ways to identify and quantitate chemical substances. Just 50 years ago, fresh from the U.S. Infantry, I returned to the University of Nebraska to start graduate research with Professor $\mathrm{H}$. Armin Pagel, a real analytical chemist. He had earned his Ph.D. under Professors I. M. Kolthoff and E. B. Sandell, authors of the authoritative textbook Quantitative Analysis. My "modern" analytical research involved the quantitative extraction of organic acids with tributyl phosphate [1], the amazing new solvent of the "Purex" process that to this day is the primary method for plutonium purification. My Cornell graduate research with Professor William T. Miller, an organofluorine chemist, did involve synthesis, but had state-of-the-art instrumentation for fractional distillation, UV spectra, and high pressure reactions. I had a great postdoctoral experience at the University of Iowa with Professor Ralph L. Shriner, author with R. C. Fuson of the classic Qualitative Organic Analysis.

In $1950 \mathrm{I}$ was introduced to mass spectrometry at the Dow Chemical Company, Midland, MI, by Victor J. Caldecourt, Jason L. Saunderson, and Norman Wright. This was in the Spectroscopy Laboratory that had world-class capabilities in infrared, Raman, NMR,
X-ray powder diffraction, emission spectroscopy, and radiochemical methods. Thanks to the tutelage of du Pont's Steve dal Nogare and ICI's H. N. Wilson, we also added gas chromatography and then gas chromatography-mass spectrometry [2]. In contrast, Dow's Analytical Laboratory had volumetric and gravimetric analysis, electrochemistry, and spectrophotometry. My forced awareness of this growing dichotomy in what should be the field of "analytical chemistry" was sharpened with my transfer to the Boston area and Dow's Eastern Research Laboratory, whose basic research mission gave me a fine incentive to become acquainted with some of the best academic chemistry departments. During those years many of these departments drastically downscaled or dropped their analytical chemistry.

This low point of the field had a bright side for me personally. Professor L. B. "Buck" Rogers was implementing revolutionary changes in the Analytical Division of the Chemistry Department at Purdue University, and in 1964 he invited me to join in this. Important evidence of how wonderful this position has been for me personally is given in Tables 1 and 2, which list my Ph.D. students and co-authors, respectively, and in this special journal issue authored by some of these people. Although I am unusually indebted to all of

Table 1. Ph.D. recipients, 1968-1995

\begin{tabular}{llll}
\hline 1968 & Edward M. Chait & 1980 & Barbara L. Atwater \\
1968 & William F. Haddon & 1982 & Michael P. Barbalas \\
1969 & Robert D. Board & 1984 & Douglas B. Stauffer \\
1969 & Richard D. Klimowski & 1985 & Paul O. Danis \\
1971 & David J. McAdoo & 1986 & I. Jonathan Amster \\
1971 & John E. Coutant & 1988 & Joseph A. Loo \\
1971 & Timothy Wachs & 1988 & Rong Feng \\
1971 & Richard A. Kornfeld & 1990 & Evan R. Williams \\
1972 & Philip Irving & 1990 & Bing-Huang Wang \\
1972 & Shih-Chuan Tsai & 1990 & Stanton Loh \\
1973 & Kain-Sze Kwok & 1991 & Mei-Yi Zhang \\
1974 & Paul F. Bente, Ill & 1991 & Kent B. Henry \\
1975 & Gail M. Pesyna & 1991 & Donald E. Drinkwater \\
1976 & Henry E. Dayringer & 1992 & Aberra Fura \\
1977 & Bobby G. Dawkins & 1994 & Russell A. Chorush \\
1978 & Frank M. Bockhoff & 1995 & Michael W. Senko \\
1980 & In Ki Mun & 1995 & Peter B. O Connor \\
1980 & Peter J. Todd & 1995 & Daniel P. Little \\
1980 & Kevin S. Haraki & & \\
\hline
\end{tabular}


Table 2. Other publication coauthors

\begin{tabular}{|c|c|c|c|}
\hline Joanne A. Gilpin & Guiseppe Innorta & Cindy L. Fisher & David H. Russell \\
\hline Mynard C. Harnming & A. J. Kresge & S. J. Abbott & Mohammad Sharaf \\
\hline Roland S. Gohlke & Bernard G. Giessner & S. R. Jones & Robert B. Spencer \\
\hline Robert C. Golesworthy & Terry E. Parks & S. A. Weinman & J. J.P. Furlong \\
\hline Jonathan W. Amy & John A. Michnowicz & Jeremy R. Knowles & Brian T. Chait \\
\hline William E. Baitinger & Peter Rogerson & Donald C. McGilvery & Frank H. Field \\
\hline Maurice M. Bursey & W. S. Trahanovsky & Kotaro Hosokawa & Frank C.-Y. Wang \\
\hline John Pinzelik & Akira Tatematsu & Earl R. Lory & Paul Klymko \\
\hline Martin Senn & Hans-Kaspar Wipf & Gregory J. Wendel & James C. Ray, Jr. \\
\hline Charles N. Reilley & Malcolm McCamish & Michael R. Wixom & Barry K. Carpenter \\
\hline Thomas W. Shannon & Karsten Levsen & T. E. Niemi & O. Zha \\
\hline Charles R. McKinney & Ikuo Sakai & Akira Hirota & Lionel Salem \\
\hline Stephen M. Kimball & Donald M. Jerina & Richard D. Bowen & Mauro Marchetti \\
\hline Setsuo Takeuchi & Michael A. Baldwin & Frank P. Pagano & Andre Maquestiau \\
\hline Roy W. Curtiss & Johann Winkler & Robert F. Pegues & Jeffrey Shabanowitz \\
\hline Rengachari Venkataraghavan & Marianne A. Busch & J. H. Vajda & Donald F. Hunt \\
\hline Nicholas J. Turro & B. A. Meyer & Alexander G. Harrison & Bernard Leyh \\
\hline D. W. Weiss & Robert C. Platt & J.G. Dillard & I. G. Brown \\
\hline William T. Pike & James W. Serum & S. R. Heller & J. C. Lorquet \\
\hline Thomas E. Mead & Richard G. Werth & G. W. A. Milne & Brigitte Leyh-Nihant \\
\hline C. Grant Warner & Patrick Arpino & Chrysotomos Wesdemiotis & B. J. Smith \\
\hline Thomas A. Bryce & Robert H. Hertel & Daniel R. Bartholomew & Leo Radom \\
\hline J. A. Nicholson & Robert D. Villwock & Michael T. Cheng & Andrea B. Twiss-Brooks \\
\hline Charles H. Jarboe & Nico M. M. Nibbering & Gary H. Kruppa & John P. Quinn \\
\hline Patricia C. Wszolek & Takao Nishishita & Frantisek Turecek & Steven I. Ryu \\
\hline James H. Brewster & Christian C. Van de Sande & Steven L. Cohen & Yuer Shi \\
\hline Michael L. Gross & Rudi Knutti & S.-C. Cheng & Detlev Suckau \\
\hline Robert B. Fairweather & Bas Van de Graaf & Katherine M. Dully & Steven C. Beu \\
\hline George E. van Lear & Paul P. Dymerski & Chuan-jie Guo & Francis M. Wampler, III \\
\hline F. Peter Boer & Richard M. Prinstein & Donald W. Peterson & Ilan Vidavsky \\
\hline William O. Perry & Claus Köppel & Steven O. Russo & Y. T. Li \\
\hline Horst D. R. Schuddemage & T. J. Broxton & D. A. Salvucci & Y. L. Hsieh \\
\hline Sixten Abrahamsson & Y. T. Pang & Werner Staedeli & Jack D. Henion \\
\hline Einar Stenhagen & John F. Smith & John L. Occolowitz & Bruce Ganem \\
\hline J. Okamoto & Stephen R. Lowry & Christopher J. Proctor & Pierre Longevialle \\
\hline H. Tsuyama & Thomas L. Isenhour & Thomas A. McCarrick & Neil L. Kelleher \\
\hline Y. Nakajima & J.B. Justice & Charles E. Hudson & J. Paul Speir \\
\hline Tomatsu Noda & Michael M. Cone & A. J. Henk Boerboom & Troy D. Wood \\
\hline H. William Major & David N. Witiak & Rosemary Csencsits & Theodore W. Thannhauser \\
\hline James S. Smith & Victor L. Tal'roze & Robert B. Ellis & Lorenzo H. Chen \\
\hline Leon J. Schiff & David D. Speck & Jacqueline A. Tso & George L. Kenyon \\
\hline K. A. Zirvi & Myung S. Kim & R. B. Cody, Jr. & Colleen A. Costello \\
\hline C. M. Schmidt & James D. Dill & J.A. Kinsinger & Tadhg P. Begley \\
\hline Ian Howe & T. E. Smith & Sahba Ghaderi & Gary A. Valaskovic \\
\hline E. B. Delany & S. R. Smith & C. E. Brown & Einar Fridriksson \\
\hline Chava Lifshitz & Robert C. Dunbar & M. E. Castro & David J. Aaserud \\
\hline
\end{tabular}

them over the years for their research productivity with me [3], I am particularly proud of their far greater accomplishments since then. I submit that these papers are worthy of your careful study not only for their fine scientific content, but also as impressive illustrations of the renaissance in analytical chemistry, again the central science of chemistry.

\section{Acknowledgments}

To reiterate, I am totally indebted to my Dow and academic co-workers for outstanding contributions in ideas, research, and instrumentation, and to generous research funding, in particular from the National Institutes of Health and the National Science Foundation.

\section{References}

1. Pagel, H. A.; McLafferty, F. W. Anal. Ch'm. 1948, 20, 272-276.

2. Gohlke, R. S.; McLafferty, F. W. I. Am Soc. Mass Spectrom. 1993, 4, 367-371.

3. McLafferty, F. W. I. Am. Soc. Mass Spectrom. 1990, 1, 1-5. 